\title{
PERCUTANEOUS INJECTION OF FIBRIN GLUE RESOLVES PERSISTENT NEPHROCUTANEOUS FISTULA COMPLICATING COLONIC PERFORATION AFTER PERCUTANEOUS NEPHROLITHOTRIPSY
}

\author{
doi: $10.1590 / \mathrm{S} 1807-59322009000700017$
}

Eduardo Paula Miranda, ${ }^{\mathrm{I}}$ Gustavo Pinto Ribeiro, ${ }^{\mathrm{II}}$ Diego Costa Almeida, ${ }^{\mathrm{I}}$ Ariel Gustavo Scafuri ${ }^{\mathrm{I}}$

\section{INTRODUCTION}

Colonic perforation (CP) is an extremely rare complication of percutaneous nephrolithotrypsy (PCNL). ${ }^{1}$ Nephrocutaneous fistulas after PCNL are unusual. Typically, they resolve with conservative measures such as observation, ureteral stenting, and, occasionally, percutaneous drainage. ${ }^{2}$ We present a case of nephrocutaneous fistula as a result of a $\mathrm{CP}$ after PCNL that was treated with percutaneous injection of fibrin glue.

\section{CASE REPORT}

A 68-year-old man, who weighed $75 \mathrm{~kg}$ and was 1.55 meters tall, presented with a left partial staghorn renal stone and was submitted to PCNL. The procedure required two puncture sites (a first failed puncture and a second uneventful posterior puncture). After successful completion of the procedure, with no residual stones, a double-J stent was advanced down the ureter, and a nephrostomy tube was left in place. On the second postoperative day, the patient developed nausea, fever, chills, and left flank pain. Blood analysis revealed total leukocyte count of $18,000 / \mu \mathrm{L}$ with predominant neutrophils. Urinalysis showed 20-30 pus cells/ field, and urine culture revealed growth of Proteus mirabilis.

During physical examination, fecal content was observed through the nephrostomy tube. Computed tomography (CT) of the upper abdomen revealed the presence of contrast in the

${ }^{\text {I }}$ Federal University of Ceará, Faculty of Medicine - Fortaleza/CE, Brazil. "I Federal University of Vale do São Francisco, Faculty of Medicine - Petrolina/PE, Brazil.

Email: mirandaep@yahoo.com.br

Tel: 55853267.2950 peritoneal cavity, causing us to suspect colonic perforation secondary to PCNL. We started the patient on antibiotics and relocated the nephrostomy tube, but symptoms failed to improve, so the patient was sent to the operating room. During the procedure, the right parietocolic recess was opened. A tiny perforation was noted and then treated with a double suture of the colon and drainage of the area.

The next day, the patient had improved considerably, and the nephrostomy tube was withdrawn, as the patient was already using a double-J stent. Upon withdrawal of the tube, high-volume urine drainage was observed, even after 4 days of continuous bladder drainage and double-J stenting.

The patient was once more taken to the operating room, and a retrograde ureteropyelography was performed, revealing an 8-mm diameter nephrocutaneous fistula (Figure 1). A guide wire was placed through the fistula under

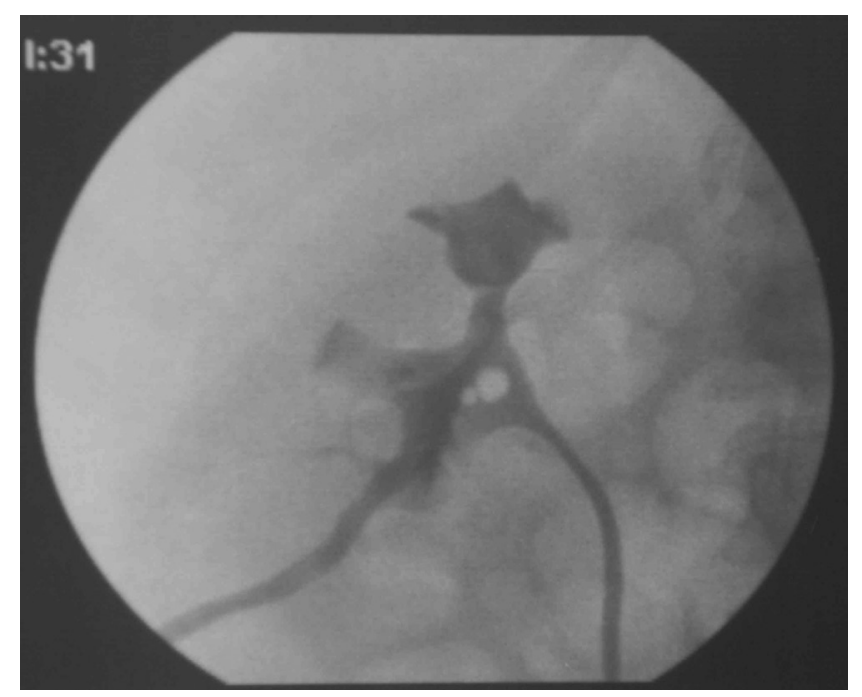

Figure 1 - Fluoroscopic image showing fistula tract 
fluoroscopic guidance. A 6.5-F catheter was advanced over the wire to the level of the kidney sheath, and a flexible fibrin glue applicator was placed through the catheter (figure 2). Approximately $5 \mathrm{ml}$ of fibrin glue was injected throughout the fistula tract as the catheter was slowly withdrawn. A control fluoroscopic image was obtained (Figure 3), revealing the absence of further communication between the renal sheath and the skin. On the first postoperative day, no urinary leakage was observed; at a four-week follow-up, the patient was asymptomatic with unremarkable findings at clinical examination. At this time, the double-J catheter was withdrawn, and no urinary leakage was seen after that.

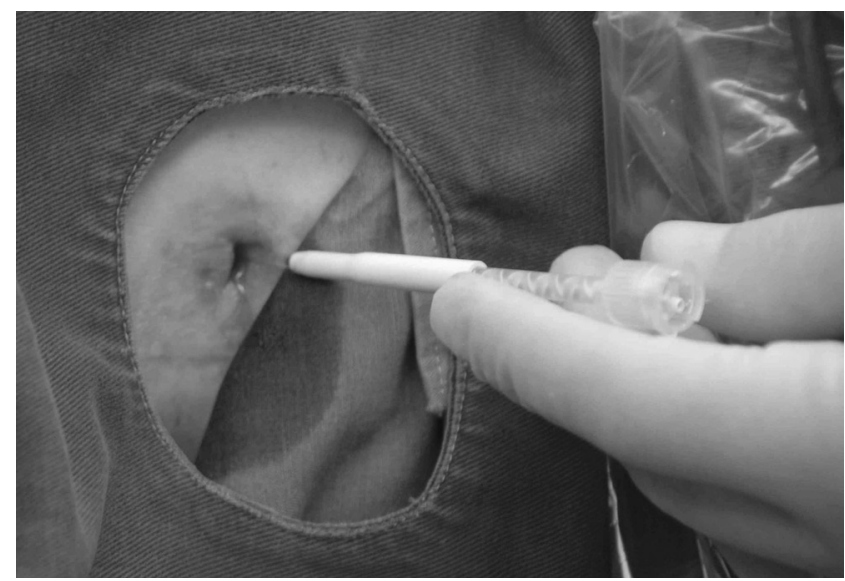

Figure 2 - Fibrin glue applicator being placed through the catheter

\section{DISCUSSION}

CP complicates $\sim 0.3 \%$ of all procedures and is considered a distressful complication. The diagnosis includes multiple symptoms and signs that occur immediately or several days after the procedure. Unrecognized colonic injury can result in abscess formation, nephrocolic or colocutaneous fistulae, peritonitis, or sepsis. ${ }^{1}$

Some factors may increase colonic injury during PCNL, such as previous intestinal bypass surgery, female sex, old age, low body weight, horseshoe kidney, and previous renal surgery. The incidence is greater on the left side, with lower calicial puncture and lateral origin of the puncture. The most important etiology for this complication is retrorenal or posterolateral position of the colon. Early diagnosis and management is the best way to avoid complications from CP. ${ }^{3,4}$

$\mathrm{CP}$ can be identified by antegrade nephrostogram, which must be performed in patients with high risk for colonic injury, who develop unexplained fever postoperatively. However, the best diagnostic tool to detect perforation of the colon by the nephrostomy tube is abdominal $\mathrm{CT} .^{4}$

The first step after diagnosing $\mathrm{CP}$ is separating the

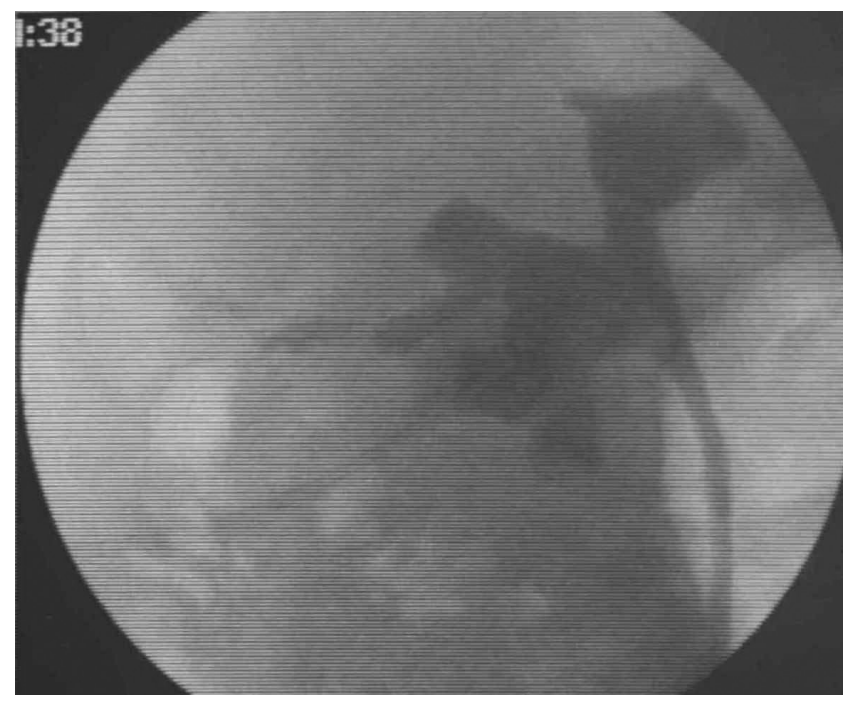

Figure 3A - Fluoroscopic image 1 minute after fibrin glue application

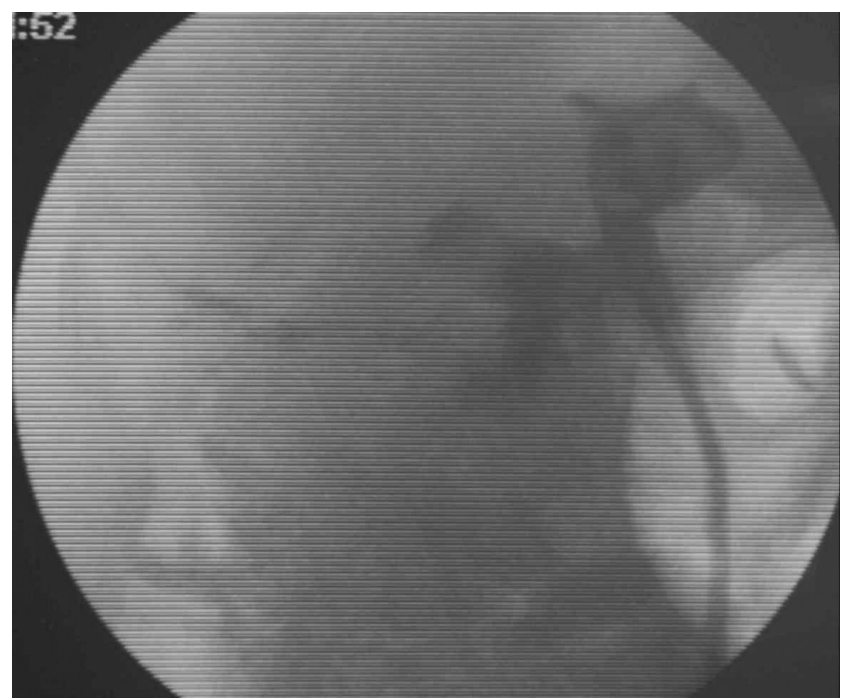

Figure 3B - Fluoroscopic image 5 minutes after fibrin glue application

nephrocolic communication, including urinary drainage with the indwelling double-J stent and removal of the nephrostomy tube from the pelvicalicial system. Conservative treatment is usually successful in patients with early-diagnosed retroperitoneal colonic injury; however, late diagnosis can result in colostomy. This observation highlights the importance of early diagnosis and treatment of colonic injury prior to the establishment of colocutaneous fistula. ${ }^{4}$

Nephrocutaneous fistula after PCNL has a reported incidence in the range of 1.8 to $21 \% .{ }^{5}$ Urine leaks can spontaneously resolve, but some require intervention in the form of ureteral stenting and/or urethral catheter placement. Urine leaks can also require percutaneous drainage or surgical correction. The use of fibrin glue for the treatment of nephrocutaneous fistula is quite novel, with only limited coverage in the literature. 
Fibrin glue can be used for several applications, such as providing support to approximating sutures in various procedures (pyeloplasty, ureteral anastomosis, vasovasostomy, and vasoepididymostomy), controlling bleeding, and preventing urinary leakage in the treatment of renal trauma. Reported uses of fibrin glue to correct fistulas have involved vesicovaginal fistulas, ureterovaginal fistulas, colovesicovaginal fistulas, vesicoperineal fistulas, colovesical fistulas, fistulas between an ileal conduit and the skin, and refractory transplant kidney-ureter fistulas. ${ }^{6}$ Other uses include the treatment of bladder injuries sustained during gynecologic procedures, traumatic ureteral injuries, prostate-rectal fistulas, and inflammatory urethrocutaneous fistulas. ${ }^{7}$ Fibrin glue can be used either as monotherapy or as a bolster over sutures. The potential complications are allergic reaction, immunologically induced coagulopathy, thromboembolic complications, and the theoretical risk of viral transmission. ${ }^{6}$

Nephrocutaneous fistula after PCNL is usually managed successfully with observation. Because our case consisted of an elderly patient complicating with a $\mathrm{CP}$, who required a recent course of antibiotics, we thought that the administration of fibrin glue would result in prompt closure of the urine leak and provide faster relief to our patient.

Fibrin glue application not only prevents the necessity of more invasive surgical correction of persistent nephrocutaneous fistulas but also represents a good initial strategy for selected cases. The optimal results we obtained and the absence of reported complications in urologic procedures lead us to recommend this therapeutic option for extensive use.

\section{REFERENCES}

1. Gerspach JM, Bellman GC, Stoller ML, Fugelso P. Conservative management of colon injury following percutaneous renal surgery. Urology. 1997;49:831-6

2. Baughman SM, Morey AF, Van Geertruyden PH, Radvany MG, Benson AE, Foley JP. Percutaneous transrenal application of fibrin sealant for refractory urinary leak after gunshot wound. J Urol. 2003;170:522-3.

3. Morse RM, Spinak JP, Resnick MI. Iatrogenic colon and rectal injuries associated with urologic intervention: report of 14 patients. J Urol. 1988;140:101-3.
4. Bradford TJ, Wolf JS. Percutaneous injection of fibrin glue for persistent nephrocutaneous fistula after partial nephrectomy. Urology. 2005;65:799.e43-799.

5. Nieder AM, Taneja SS: The role of partial nephrectomy for renal cell carcinoma in contemporary practice. Urol Clin North Am. 2003;30:52942.

6. Shekarriz B, Stoller ML. The use of fibrin sealant in urology. J Urol. 2002;167:1218-25.

7. Evans LA, Ferguson KH, Foley JP, Rozanski TA, Morey AF. Fibrin sealant for the management of genitourinary injuries, fistulas and surgical complications. J Urol. 2003; 169:1360-62. 